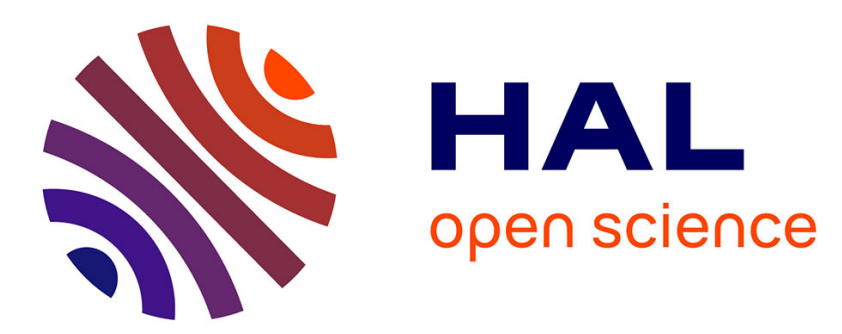

\title{
Application of a photothermal displacement technique for the characterization of diamond-like carbon films
}

\author{
G. Amato, G. Benedetto, L. Boarino, M. Maringelli, R. Spagnolo
}

\section{To cite this version:}

G. Amato, G. Benedetto, L. Boarino, M. Maringelli, R. Spagnolo. Application of a photothermal displacement technique for the characterization of diamond-like carbon films. Journal de Physique IV Proceedings, 1994, 04 (C7), pp.C7-237-C7-240. 10.1051/jp4:1994756 . jpa-00253285

\section{HAL Id: jpa-00253285 https://hal.science/jpa-00253285}

Submitted on 1 Jan 1994

HAL is a multi-disciplinary open access archive for the deposit and dissemination of scientific research documents, whether they are published or not. The documents may come from teaching and research institutions in France or abroad, or from public or private research centers.
L'archive ouverte pluridisciplinaire HAL, est destinée au dépôt et à la diffusion de documents scientifiques de niveau recherche, publiés ou non, émanant des établissements d'enseignement et de recherche français ou étrangers, des laboratoires publics ou privés. 


\title{
Application of a photothermal displacement technique for the characterization of diamond-like carbon films
}

\author{
G. Amato, G. Benedetto, L. Boarino, M. Maringelli and R. Spagnolo \\ Istituto Elettrotecnico Nazionale Galileo Ferraris, Strada delle Cacce 91, 10135 Turin, Italy
}

\begin{abstract}
The paper presents preliminary results of a study about the possibility to determine the thermoelastic properties of diamond-like carbon films by means of a photothermal deflection technique. The technique allows to perform measurements as a function of temperature in the range $10-300 \mathrm{~K}$ The results are in qualitative agreement with theoretical predicitons.
\end{abstract}

\section{INTRODUCTION}

Photothermal displacement technique (PD) [1] has been widely applied in past years for the study of material properties. The main advantage of the technique with respect to other photothermal techniques is its capability to perform measurements in vacuum and, therefore, at low temperatures. In particular the technique has recently been used to investigate the thermal properties of semiconductors at room temperature [2] and to determine the variation of thermal conductivity versus temperature in silicon [3]. Also in the case of thin films deposited on a substrate, the technique has been applied to the measurement of thin film thickness and the possiblity to separate the contribution of the film from that of the substrate has been indicated [4].

On the other hand diamond-like carbon (DLC) films are successfully used because of their mechanical and thermal properties, as coatings in optical components and in abrasive and turning tools [5]. Whilst the mechanical properties of DLC films have been extensively investigated over the past years [6], there is a lack of experimental data on thermal and thermoelastic properties of these films. Thermal properties of diamond and diamond like carbon films are difficult to measure owing to the high thermal conductivity of these films. The general approach is to confine the heat flow to the film by removing the substrate. Among the different techniques used for thermal characterization of diamond materials, thermal wave/mirage effect measurements have been suggested as a conctactless method, usable also for in situ characterization[7].

Moreover, it must be underlined that thermal properties of DLC film are important from another point of view, that is to give information on the microstructure, impurity content and double bonds in the film. For instance it has been demonstrated that the presence of $\mathrm{sp} 2$ bonding decreases the thermal conductivity [8]. This kind of information seems to be even more useful if measurements are performed as a function of temperature. The paper presents the preliminary results obtained by the application of a PD technique on DLC films deposited by different methods on a silicon substrate in the temperature range 30-300 K. 


\section{EXPERIMENTS}

The basic principle of PD technique is well known (see Fig. 1). A modulated laser beam impinges over a sample; the absorbed light is converted into heat by nonradiative deexcitation processes. The sample expands as its temperature rises and the thermoelastic deformation of the surface is detected through the variation of the reflection angle of a probe laser beam. In the experiment described here [3] heating is provided by an $\mathrm{Ar}^{+}$ion laser beam (514-nm) mechanically chopped at $65 \mathrm{~Hz}$, with a power of $100 \mathrm{~mW}$ focused in a $100 \mu \mathrm{m}$ spot. The probe beam is a He-Ne laser $(633 \mathrm{~nm})$, has a power of $5 \mathrm{~mW}$ and is focused to $50 \mu \mathrm{m}$. The slope of the surface displacement is measured by a position-sensitive detector (through the deflection of the reflected probe beam).

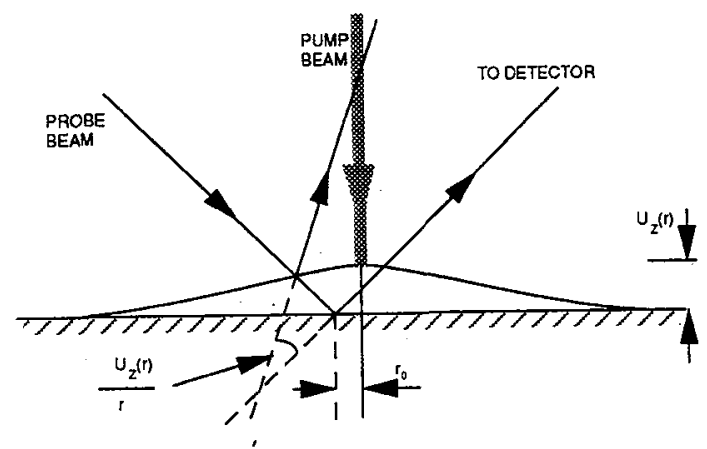

Fig.1 Schematic representation of PD technique

The investigated samples were deposited by different techniques, in particular samples 1-3 were prepared in a RF sputtering system and samples $4-6$ by plasma enhanced chemical vapour deposition. All the samples were deposited on a floating-zone (FZ) crystalline silicon substrate. Each sample was placed in the experimental chamber of a continuous He flow cryostat. Optical access was through vacuum sealed quartz windows. The sample temperature was accurately measured with a thin-film thermometer gauge attached to the sample holder. The relative distance between the pump and probe beam could be varied in the range $0-500 \mu \mathrm{m}$ by means of a translation stage.

\section{RESULTS AND DISCUSSION}

The measurement procedure is similar to that described in ref.[3], that is to perform measurements at a fixed temperature as a function of distance and at a fixed distance, in correspondence of the maximum of the PD signal, as a function of temperature. For bulk samples of doped silicon it has been demonstrated [3] that this method allows to determine the variation of thermal conductivity as a function of temperature. In fact, in the case of homogeneous samples, for a given experimental configuration (that is dimensions and power of the heating beam, sample thickness and Poisson's ratio), the PD signal amplitude depends on the thermal expansion coefficient, thermal conductivity and thermal diffusion length. The relation between PD signal and thermal expansion coefficient is of direct proportionality. On the other side, the relation between PD signal and thermal conductivity is through a factor, independent on the distance $r_{0}$ between the excitation and probe beam, and an integral in which the thermal conductivity is contained in the thermal diffusivity. It can be demonstrated that the dependence of the signal on $r_{0}$ is predominantly governed by lateral heat diffusion.

In the case of a layered structure the problem is more complex. The contribution of the thin film deformation to the total deformation, as can be deduced from relationship (37) of ref.[4], is essentially connected to the ratio between the thermal expansion coefficients of film and substrate. In the case under study the contribution of the film should be particularly evident in the temperature zone in which the . 


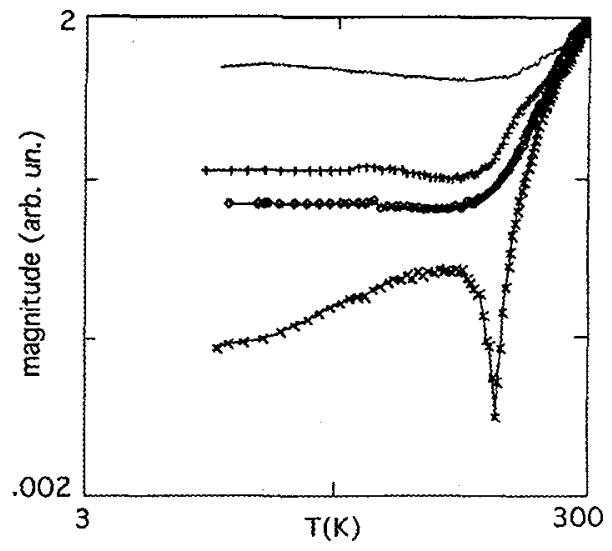

Fig. 2. Temperature variation of PD signal, xxx: uncoated silicon sample, +++ : sample 2, 000 : sample 3 .

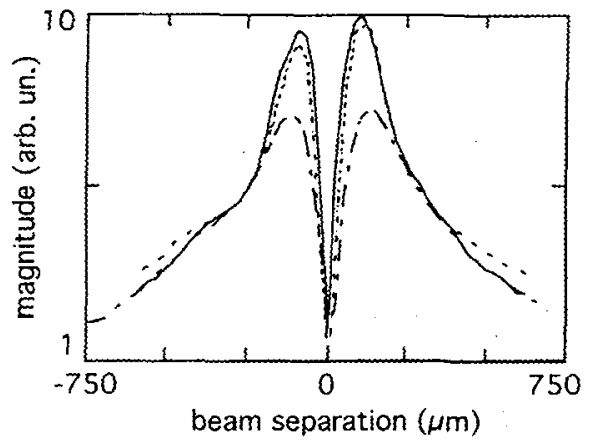

Fig. 3. PD signal as a function of beam separation, — : sample $1, \ldots$ : uncoated silicon, - - : : sample 2.

thermal expansion coefficient of substrate (silicon) crosses zero $(110-130 \mathrm{~K})$. Outside this zone, the signal amplitude is also related to the thermal conductivity values of the films

Fig.2 reports the temperature variation of PD signal for an uncoated silicon sample and for samples 1-3. For the uncoated silicon sample, the signal behaviour is characterized by a change of sign in correspondence to the value of zero crossing in the curve of the thermal expansion coefficient, as foreseen by the theory [1]. The presence of the film is evident in the other curves. It can be noticed that the lower curve corresponds to sample 3, which among the films deposited by sputtering is the one with highest hydrogen content $(30 \%)$.

Fig. 3 reports the variation of PD signal as a function of the distance between the pump and the probe beam at room temperature for the uncoated sample and for samples 1 and 2. For all curves the slope gradually increases when the pump beam comes into the vicinity of the probe beam, than goes sharply to zero at the center of the overlap and roughly repeats itself when $r_{0}$ changes sign (the slight difference of form between the two parts of the curves is related to experimental conditions). For bulk materials, as uncoated silicon, the shape of the curve as a function of the distance is mainly related to the thermal diffusivity of the material. The thermal diffusivity can be determined by a best fit procedure between experimental data and theoretical model (relationships (6) and (7) of ref. [1]). A value of $0.8 \mathrm{~cm}^{2} / \mathrm{s}$ was obtained for silicon. The procedure of the fit is described in detail in ref. [3]. The curves relative to samples 1 and 2 show a different behaviour, at low and high distances. At lower distances, the presence of the film is evident; at higher distances the signal approximates that of the uncoated silicon. An estimation (according to the method described in ref. [3]), based on the assumption that lateral heat diffusion dominate the thermoelastic response, gives thermal diffusivity values of $1.2 \mathrm{~cm}^{2} / \mathrm{s}$ for sample 1 and $2.5 \mathrm{~cm}^{2} / \mathrm{s}$ for sample 2, which are reasonable if compared to the values of thermal diffusivity of amorphous carbon given in the literature. However, the contribution of heat diffusion in the substrate cannot be neglected.

In order to better study the contribution of the thin film deformation to the total signal, fig. 4 and 5 report the ratios between the signal measured on DLC samples, A, and the signal of the uncoated silicon sample, $A_{S i}$, vs. temperature; fig. 4 is relative to samples $1-3$, and fig. 5 to samples $4-6$. The values of the ratio are normalized to 1 at room temperature for all the samples. The curves are characterized by a peak in the temperature zone were the value of the thermal expansion coefficient of silicon is near to zero, an experimental confirm of the importance of the ratio between the thermal expansion coefficient of film 


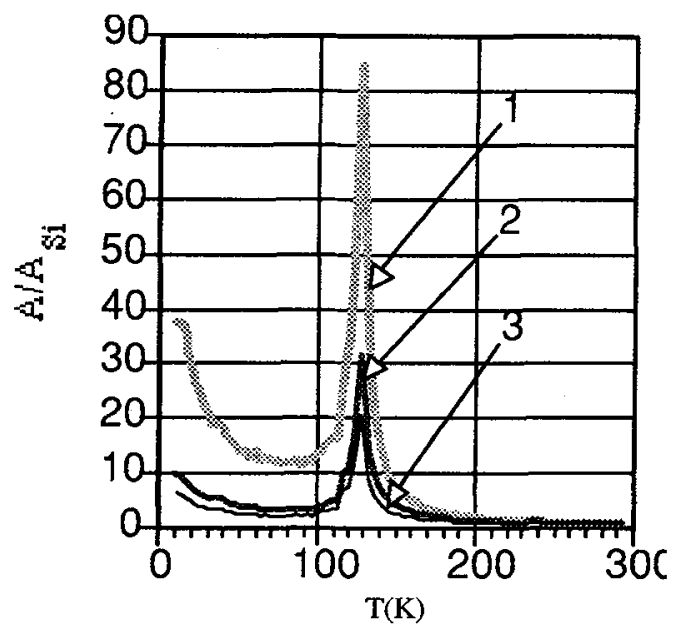

Fig.4. Ratio between experimental PD signals of film and silicon substrate for samples 1-3.

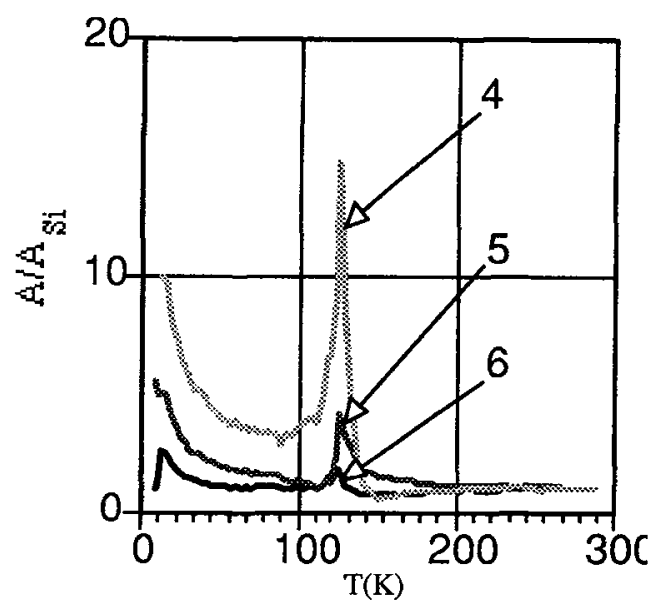

Fig.5. Ratio between experimental PD signals of film and silicon substrate for samples 4-6.

and substrate to the total $\mathrm{PD}$ signal. Moreover, the values of $\mathrm{A} / \mathrm{A}_{\mathrm{Si}}$ are much larger for the samples deposited by sputtering than for the samples deposited by plasma enhanced chemical vapour deposition. This can be interpreted as due to the fact that the former deposition technique gives samples with values of $\mathrm{sp} 2 / \mathrm{sp} 3$ higher than the latter, that is films where the two-dimensional graphite-like structure is predominant with respect to three-dimensional diamond-like network. A further consideration is that for films deposited by the same technique, the $A / A_{S i}$ values are correlated to the hydrogen content, with higher values corresponding to higher hydrogen content. The dependence of the thermal expansion coefficient of thin films of amorphous silicon on the hydrogen content has been evidenced in [9].

In conclusion, it has been confirmed that the PD technique can be applied for the characterization of thermal and thermoelastic properties of DLC films as a function of temperature. While in the case of bulk samples, under the hypothesis that the values of thermal expansion coefficient and specific heat are known, the technique allows to quantitative determine the variation of thermal conductity versus temperature [3], in the case of thin film deposited on a substrate the interpretation of the experimental results is more complicate. However these preliminary results are in good qualitative agreement with the theoretical predicitons. The quantitative evaluation of the thermal conductivity, thermal diffusivity and thermal expansion coefficient require further investigation.

\section{References}

[1]Olmstead M.A.,Amer N.M.,Kohn S.,Fournier D. and Boccara A.C.,Apply. Phys. A 32(1983) 141-154.

[2]Kuo B.S.W., Li J.C. and Schmid A.W., Apply. Phys. A 55(1992) 289-296.

[3]Benedetto G., Boarino L. and Spagnolo R., Rev. Sci. Instrum. 64(1993) 2229-2232.

[4]Welsch E., J. Mod. Optics 38(1991) 2159-2176.

[5]Angus J.C. and Hayman C.C., Science 241(1988) 913-921.

[6]Robertson J., Phys.Rev.Lett, 68(1992) 220-223.

[7]Pryor R.W., Lanhua Wei, Kuo P.K. and Thomas R.L., in Diamond and Diamond-Like Films and

Coatings, NATO ASI Series, Series B:Physics, Vol.266 (1991) pp. 723-728.

[8]Anthony T.R., Phil.Trans.R.Soc.Lond.A 342(1993) 245-251.

19|Jancen F., Machonkin M.A., Palmieri N. and Kuhman D., J.Appl.Phys. 62(1987) 4732-4736. 